\title{
1,4-Dipolar cycloaddition in organic synthesis: a facile route to isoquinoline fused heterocycles
}

\author{
Vijay Nair,* Anakkalil Ramachandran Sreekanth, Narayana Pillai Abhilash, Akkattu \\ Thankappan Nair Biju, Luxmi Varma, Sreemathi Viji, and Saumini Mathew \\ Organic Chemistry Division, Regional Research Laboratory (CSIR), Trivandrum-695019, India \\ E-mail: vijaynair2001@yahoo.com
}

\section{Dedicated with best wishes to Professor S. Swaminathan on the occasion of his $80^{\text {th }}$ birthday}

(received 16 Nov 04; accepted 08 Jan 05; published on the web 17 Jan 05)

\begin{abstract}
The three component condensation reactions involving isoquinoline, dimethyl acetylenedicarboxylate and carbonyl dipolarophiles such as $o$ - and $p$-benzoquinones and $N$ substituted isatins constitute a one-pot synthesis of a variety of [1,3] oxazino isoquinoline derivatives via 1,4-dipolar cycloaddition.
\end{abstract}

Keywords: Dimethyl acetylenedicarboxylate, $N$-substituted isatins, isoquinoline, $o$ - and $p$ benzoquinones

\section{Introduction}

In addition to the well known hetero-Diels-Alder reactions, ${ }^{1}$ 1,4-dipolar cycloaddition constitutes a potentially versatile process for the construction of six membered heterocycles. The basic principles of 1,4-dipolar cycloaddition were provided by the pioneering work of Huisgen and coworkers. ${ }^{2}$ Noteworthy developments in this area have been the introduction of heteroaromatic betaines as 1,4-dipoles ${ }^{3}$ and the utilization of 1,4-dipole equivalents in formal 1,4-dipolar cycloaddition reactions. ${ }^{4}$

Apart from a few isolated reports in the literature, ${ }^{5}$ the potential of "Huisgen 1,4-dipoles" for the construction of various six membered heterocycles remains underexploited. An interesting example of this type is the dipole $\mathbf{1}$ generated from isoquinoline and dimethyl acetylenedicarboxylate (DMAD), whose existence was confirmed by Huisgen ${ }^{6}$ (Figure 1). 


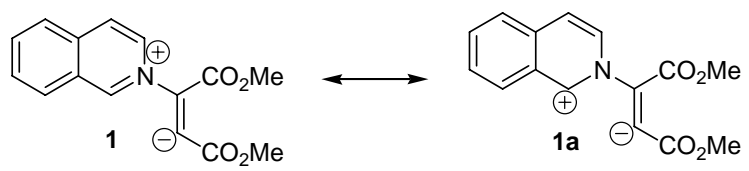

\section{Figure 1}

In view of our recent interest in developing novel multicomponent reactions for heterocyclic synthesis via dipolar intermediates ${ }^{7}$ we were intrigued by the possibility of trapping the dipole $\mathbf{1}$ with various dipolarophiles. Investigations carried out in this context have demonstrated that the dipole 1 can be effectively trapped by $N$ - tosylimines resulting in the diastereoselective synthesis of $2 \mathrm{H}$-pyrimido[2,1-a]isoquinolines. ${ }^{8 \mathrm{a}}$ Our preliminary results from studies using quinones as dipolarophiles have shown that the reaction leads to a one-pot synthesis of spiro[1,3]oxazino[2,3a]isoquinolines. ${ }^{8 b}$ In this paper we disclose the results of our extended investigations on the reactivity of dipole 1 towards various carbonyl dipolarophiles; the reactions constitute a facile route to isoquinoline fused heterocycles, which are interesting from the standpoint of their potential biological activity.

\section{Results and Discussion}

\section{Reaction of isoquinoline and DMAD with 1,2- and 1,4-benzoquinones}

Against the background presented above and in the context of our general interest in the dipolarophilic profile of quinonoid compounds, ${ }^{9}$ we examined the possibility of trapping the 1,4 dipole 1 with $o$ - and $p$-benzoquinones.

In a pilot experiment, it was observed that a mixture of 3-methoxy-4,6-bis(1,1diphenylmethyl)-1,2-benzoquinone 4 and DMAD at room temperature in anhydrous DME, when treated with isoquinoline afforded the spiro[1,3] oxazino[2,3-a]isoquinoline derivatives $\mathbf{5}$ and $\mathbf{6}$ in 91\% yield in the ratio 2:1 (Scheme 1).



\section{Scheme 1}


The products 5 and $\mathbf{6}$ were characterized by spectroscopic techniques. The IR spectrum showed strong absorptions at 1742, 1708 and $1667 \mathrm{~cm}^{-1}$ indicating the presence of ester and enone carbonyls. In the ${ }^{1} \mathrm{H}$ NMR spectrum of 5, signals due to the three methoxy groups were visible at $\delta 3.94,3.54$ and 3.40; the corresponding signals for 6 were observed at $\delta 3.90,3,64$ and 3.47. The ring junction proton of 5 was discernible as a singlet at $\delta 6.50$; the corresponding signal for 6 was seen as singlet at $\delta 6.68$. In ${ }^{13} \mathrm{C}$ NMR spectrum of $\mathbf{5}$, the characteristic signal for the spirocarbon was observed at $\delta 78.2$, whereas in the spectrum of $\mathbf{6}$, it was discernible at $\delta$ 80.9. The signals corresponding to ester and enone carbonyls of 5 were seen at $\delta 163.4,163.5$ and 194.9 and those for 6 were visible at $\delta 163.4,164.2$ and 193.1. Finally the structure and stereochemistry of the product 6 was unambiguously established by single crystal X-ray analysis.

Similar reactivity was also observed with 1,4-benzoquinones. Thus 2,5-dimethyl-1,4benzoquinone 7 when treated with DMAD in presence of isoquinoline gave $76 \%$ of the spiro[1,3] oxazino[2,3-a] isoquinoline derivative 8 (Scheme 2).

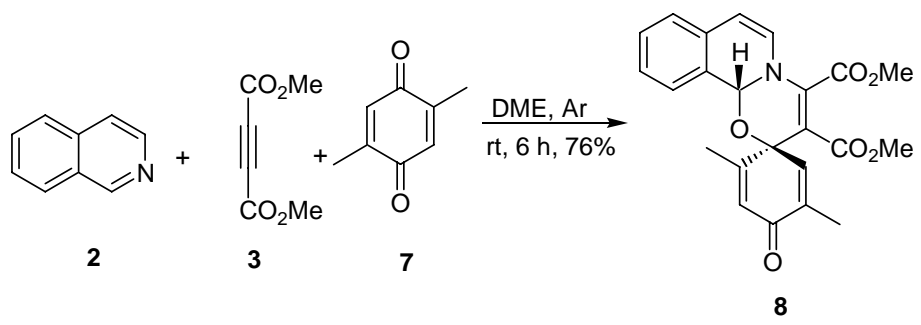

\section{Scheme 2}

Analogous results were obtained with a number of other quinones and the results are summarised in Table 1. Mechanistically the reaction can be considered to proceed via the initial formation of the 1,4-dipolar intermediate 1 from isoquinoline and DMAD, followed by its trapping with quinone carbonyl in a cycloaddition mode to give the corresponding spiro[1,3] oxazino isoquinolines (Scheme 3).

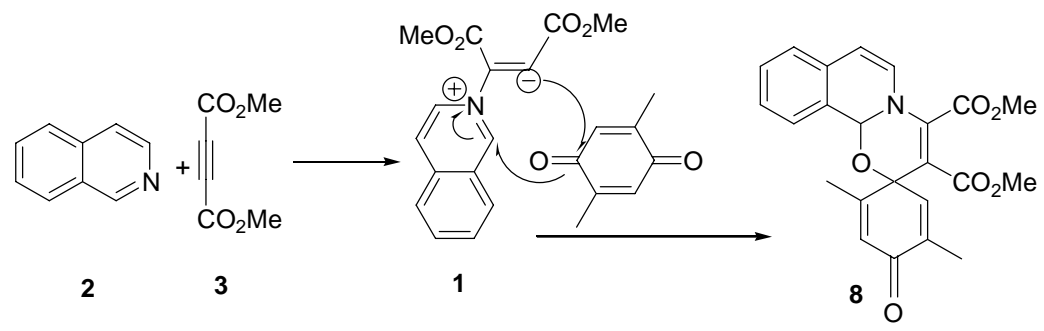

\section{Scheme 3}


Table 1. Reaction of isoquinoline and DMAD with quinines

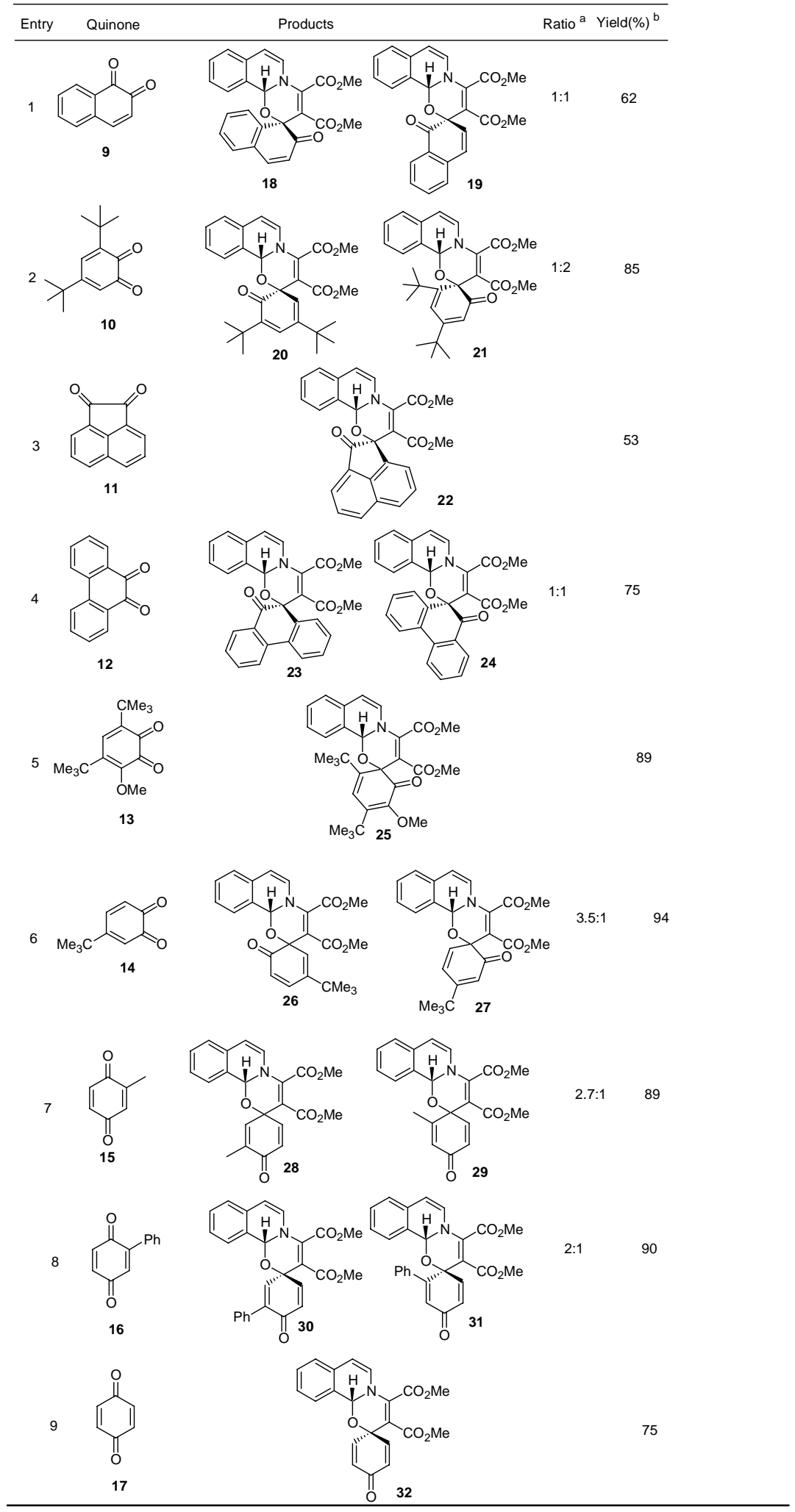

Reaction conditions $=\mathrm{DME}, \mathrm{Ar}, \mathrm{rt}, 6 \mathrm{~h},{ }^{\mathrm{a}}$ ratio of isomers. ${ }^{\mathrm{b}}$ isolated yield . 


\section{Reaction of Isoquinoline and DMAD with $N$-substituted Isatins}

Impressed by the reactivity of the dipole 1 towards quinones, it was interesting to explore its reactivity towards 1,2-diones such as $N$-substituted isatins. Thus the reaction of $N$-substituted isatins with DMAD and isoquinoline afforded the spiro[1,3]oxazino[2,3-a]isoquinoline derivatives 33-35 in moderate yields (Scheme 4).

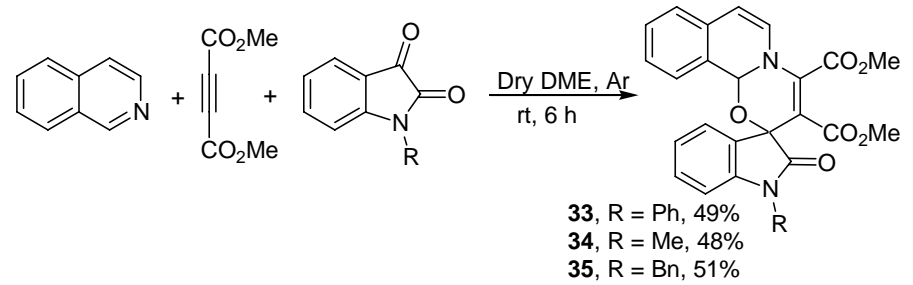

\section{Scheme 4}

he products were purified by chromatography and characterized by spectroscopic analysis. The IR spectrum of $\mathbf{3 3}$ showed the characteristic ester carbonyl absorptions at 1741 and 1711 $\mathrm{cm}^{-1}$. The amide carbonyl absorption was seen at $1620 \mathrm{~cm}^{-1}$. In the ${ }^{1} \mathrm{H}$ NMR spectrum, signals due to the methoxy groups were observed as singlets at $\delta 3.98$ and 3.53 whereas the olefinic protons were visible as doublets at $\delta 6.42(J=7.73 \mathrm{~Hz})$ and $5.83(J=7.74 \mathrm{~Hz})$. In the ${ }^{13} \mathrm{C} \mathrm{NMR}$ spectrum, the three resonance signals corresponding to the ester and amide carbonyls were seen at $\delta 162.9,163.1$ and 173.3. The signal due to the spirocarbon was discernible at $\delta 79.1$. All the other signals were also in agreement with the assigned structure.

\section{Conclusions}

In conclusion, we have developed some novel and interesting three component condensation reactions via 1,4-dipolar cycloaddition, affording a facile entry into a variety of isoquinoline fused heterocycles. In this context, it is noteworthy that these isoquinoline derivatives are known to possess interesting biological activities. ${ }^{10}$

\section{Experimental Section}

General Procedures. Melting points were recorded on a Buchi melting point apparatus and are uncorrected. NMR spectra were recorded at $300\left({ }^{1} \mathrm{H}\right)$ and $75\left({ }^{13} \mathrm{C}\right) \mathrm{MHz}$ on a Bruker DPX-300 MHz NMR spectrometer. The spectra were run in $\mathrm{CDCl}_{3}-\mathrm{CCl}_{4}$, v/v 3:1 and chemical shifts are reported $(\delta)$ relative to TMS $\left({ }^{1} \mathrm{H}\right)$ and $\mathrm{CDCl}_{3}\left({ }^{13} \mathrm{C}\right)$ as the internal standards. Mass spectra were recorded under EI/HRMS (at 5000) resolution using an Auto Spec. M mass spectrometer. IR spectra were recorded on a Nicolet Impact 400D FT-IR spectrophotometer. Elemental analyses 
were obtained on a Perkin-Elmer-2400 elemental analyzer. Dimethyl acetylenedicarboxylate was purchased from Aldrich Chemical Co. and was used without further purification.

Experimental procedure for the preparation of [1,3]oxazino[2,3-a]isoquinoline derivatives. To a stirred solution of dimethyl acetylenedicarboxylate (1 equiv) and quinone (1 equiv) in dry DME $(10 \mathrm{~mL})$ under an argon atmosphere, was added isoquinoline (1 equiv) and the reaction mixture was stirred for $6 \mathrm{~h}$ at room temperature. The solvent was then removed under vacuum and the residue on chromatographic separation on silica gel using hexane- ethyl acetate (80:20) gave spiro[1,3]oxazino[2,3-a]isoquinoline derivatives.

\section{Dimethyl-2-methoxy-3,5-(dibenzhydryl)-6-oxospiro[cyclohexa-2,4-diene-1,2'-[2H,11bH]} [1,3]oxazino[2,3-a]isoquinoline]-3',4'-dicarboxylate (5). Yellow crystalline solid; mp 208$210^{\circ} \mathrm{C} . I R(\mathrm{KBr}) v_{\max }: 2948,1742,1708,1667,1600,1499,1431,1276,1236,1148 \mathrm{~cm}^{-1} .{ }^{1} \mathrm{H}$ NMR: $\delta 7.28-6.88(\mathrm{~m}, 21 \mathrm{H}), 6.72(\mathrm{~d}, J=7.66 \mathrm{~Hz}, 2 \mathrm{H}), 6.50(\mathrm{~s}, 1 \mathrm{H}), 6.39(\mathrm{~s}, 1 \mathrm{H}), 6.34(\mathrm{~d}, J=$ $7.67 \mathrm{~Hz}, 1 \mathrm{H}), 5.84-5.72(\mathrm{~m}, 2 \mathrm{H}), 5.55(\mathrm{~s}, 1 \mathrm{H}), 5.49(\mathrm{~s}, 1 \mathrm{H}), 3.94(\mathrm{~s}, 3 \mathrm{H}), 3.54(\mathrm{~s}, 3 \mathrm{H}), 3.40$ (s, $3 \mathrm{H}) .{ }^{13} \mathrm{C}$ NMR: $\delta$ 194.9, 163.6, 163.5, 155.9, 148.0, 143.2, 141.0, 134.6, 129.9, 129.5, 129.3, $129.1,129.0,128.9,128.5,128.5,128.3,128.2$, 128.1, 126.5, 126.4, 126.3, 123.3, 105.1, 104.6, 78.2, 61.6, 53.3, 51.6, 48.9, 47.9.Anal. Calcd. for $\mathrm{C}_{48} \mathrm{H}_{39} \mathrm{NO}_{7} ; \mathrm{C}, 77.72 ; \mathrm{H}, 5.30$; N, 1.89; Found C, 77.52; H, 5.19; N, 1.78 .

Dimethyl-3-methoxy-4,6-(dibenzhydryl)-2-oxospiro[cyclohexa-2,4-diene-1,2'-[2H,11bH] [1,3]oxazino[2,3-a]isoquinoline]-3',4'-dicarboxylate (6). Yellow crystalline solid; mp 198$200{ }^{\circ} \mathrm{C}$. IR(KBr) $v_{\max }: 2955,1742,1708,1667,1600,1499,1431,1229,1148 \mathrm{~cm}^{-1} .{ }^{1} \mathrm{H}$ NMR: $\delta$ 7.30-6.95 (m, 22H), 6.80-6.78 (m, 2H), $6.68(\mathrm{~s}, 1 \mathrm{H}), 6.34(\mathrm{~d}, J=7.48 \mathrm{~Hz}, 2 \mathrm{H}), 5.74(\mathrm{~d}, J=7.76$ $\mathrm{Hz}, 1 \mathrm{H}), 5.62(\mathrm{~s}, 1 \mathrm{H}), 5.33(\mathrm{~s}, 1 \mathrm{H}), 3.90(\mathrm{~s}, 3 \mathrm{H}), 3.64(\mathrm{~s}, 3 \mathrm{H}), 3.47(\mathrm{~s}, 3 \mathrm{H}) .{ }^{13} \mathrm{C}$ NMR: $\delta 193.1$, $164.2,163.4,158.9,142.4,142.3,135.1,129.4,129.0,128.9,128.8,128.6,128.5,128.1,128.0$, 127.8, 126.7, 126.6, 126.2, 126.1, 126.0, 124.1, 108.1, 104.7, 80.9, 62.1, 53.2, 48.9, 48.1.Anal. Calcd. for $\mathrm{C}_{48} \mathrm{H}_{39} \mathrm{NO}_{7} ; \mathrm{C}, 77.72 ; \mathrm{H}, 5.30 ; \mathrm{N}, 1.89$; Found C, 77.59; H, 5.16; N, 1.82 .

Dimethyl-2,5-dimethyl-4-oxospiro[cyclohexa-2,4-diene-1,2'-[2H,11bH] [1,3]oxazino[2,3a]isoquinoline]-3',4'-dicarboxylate (8). Yellow crystalline solid; mp 168-170 ${ }^{\circ} \mathrm{C}$. $\mathrm{IR}(\mathrm{KBr}) v_{\max }$ : 2955, 1742, 1721, 1681, 1647, 1593, 1566, 1438, 1283, 1236, $1148 \mathrm{~cm}^{-1}$. ${ }^{1} \mathrm{H}$ NMR: $\delta 7.33-7.24$ (m, 3H), $7.13(\mathrm{t}, J=8.10 \mathrm{~Hz}, 1 \mathrm{H}), 6.89(\mathrm{~s}, 1 \mathrm{H}), 6.39(\mathrm{dd}, J=7.68,14.15 \mathrm{~Hz}, 1 \mathrm{H}), 6.25-6.19$ (m, $1 \mathrm{H}), 6.06(\mathrm{~s}, 1 \mathrm{H}), 5.83(\mathrm{q}, J=7.74 \mathrm{~Hz}, 1 \mathrm{H}), 3.94(\mathrm{~s}, 3 \mathrm{H}), 3.61(\mathrm{~s}, 3 \mathrm{H}), 1.96(\mathrm{~s}, 3 \mathrm{H}), 1.83(\mathrm{~s}, 3 \mathrm{H})$. ${ }^{13} \mathrm{C}$ NMR: $\delta$ 186.1, 163.9, 163.2, 156.3, 153.7, 143.6, 138.6, 134.6, 129.7, 129.0, 127.5, 127.2, 125.0, 125.3, 123.6, 109.9, 104.9, 104.6, 79.3, 53.3, 52.0, 17.4, 15.6. Anal. Calcd. for $\mathrm{C}_{23} \mathrm{H}_{21} \mathrm{NO}_{6} ; \mathrm{C}, 67.80 ; \mathrm{H}, 5.20 ; \mathrm{N}, 3.44$; Found C, 67.52; H, 5.19; N, 3.82.

Dimethyl-2-oxo-spiro[naphthylene-1,2'-[2H,11bH][1,3]oxazino[2,3a]isoquinoline] ～-3',4'dicarboxylate (18). Yellow crystalline solid; mp 165-167 ${ }^{\circ} \mathrm{C}$. IR(KBr) $v_{\max }$ : 2955, 1739, 1708, 1679, 1594, 1565, 1431, 1277, 1236, $1148 \mathrm{~cm}^{-1} .{ }^{1} \mathrm{H}$ NMR: $\delta 8.12$ (d, $\left.J=7.60 \mathrm{~Hz}, 1 \mathrm{H}\right), 7.56$ (t, $J$ $=7.48 \mathrm{~Hz}, 1 \mathrm{H}), 7.39(\mathrm{t}, J=7.52 \mathrm{~Hz}, 1 \mathrm{H}), 7.25-7.06(\mathrm{~m}, 5 \mathrm{H}), 6.92(\mathrm{~s}, 1 \mathrm{H}), 6.71(\mathrm{~d}, J=9.77 \mathrm{~Hz}$, $1 \mathrm{H}), 6.36(\mathrm{~d}, J=7.73 \mathrm{~Hz}, 1 \mathrm{H}), 5.81-5.76(\mathrm{~m}, 2 \mathrm{H}), 3.97(\mathrm{~s}, 3 \mathrm{H}), 3.44(\mathrm{~s}, 3 \mathrm{H}) .{ }^{13} \mathrm{C}$ NMR: $\delta 196.2$, $163.9,163.5,144.3,136.9,134.6,132.9,130.4,129.7,129.4,129.3,128.9,128.3,128.1,127.7$, 
127.6, 127.3, 127.1, 126.2, 125.1, 123.1, 108.3, 104.9, 79.3, 53.3, 51.5. HRMS (EI) for $\mathrm{C}_{25} \mathrm{H}_{19} \mathrm{NO}_{6}$; Calcd. 429.1212; Found 429.1139.

Dimethyl-6-oxo-spiro[naphthylene-1,2'-[2H,11bH][1,3]oxazino[2,3a]isoquinoline] -3',4'dicarboxylate (19). Yellow crystalline solid; mp 170-172 ${ }^{\circ} \mathrm{C}$. IR(KBr) $v_{\max }$ : 2955, 1742, 1708, 1667, 1598, 1570, 1430, 1320, 1277, $1149 \mathrm{~cm}^{-1} .{ }^{1} \mathrm{H}$ NMR: $\delta 7.44-7.03(\mathrm{~m}, 9 \mathrm{H}), 6.84(\mathrm{~s}, 1 \mathrm{H})$, $6.41(\mathrm{~d}, J=7.72 \mathrm{~Hz}, 1 \mathrm{H}), 6.24(\mathrm{~d}, J=9.93 \mathrm{~Hz}, 1 \mathrm{H}), 5.79$ (d, $J=7.74 \mathrm{~Hz}, 1 \mathrm{H}), 3.99$ (s, 3H), 3.46 (s, 3H). ${ }^{13} \mathrm{C}$ NMR: $\delta$ 196.7, 163.5, 163.3, 144.9, 144.7, 140.3, 130.6, 130.3, 129.6, 129.5, 129.2, 128.7, 127.6, 127.0, 126.8, 125.7, 124.9, 123.4, 123.1, 109.3, 104.8, 78.3, 53.1, 51.4. HRMS (EI) for $\mathrm{C}_{25} \mathrm{H}_{19} \mathrm{NO}_{6}$; Calcd. 429.1212; Found 429.1255

Dimethyl-3,5-(t-butyl)-6-oxospiro[cyclohexa-2,4-diene-1,2'-[2H,11bH] [1,3]oxazino[2,3a]isoquinoline]-3',4'-dicarboxylate (20). Yellow semi-solid. IR(film) $v_{\max }$ : 2955, 1742, 1708, 1667, 1593, 1566, 1430, 1276, 1229, $1148 \mathrm{~cm}^{-1} .{ }^{1} \mathrm{H}$ NMR: $\delta 7.25-7.20(\mathrm{~m}, 3 \mathrm{H}), 7.06(\mathrm{~d}, J=$ $7.37 \mathrm{~Hz}, 1 \mathrm{H}), 6.89-6.84(\mathrm{~m}, 2 \mathrm{H}), 6.35(\mathrm{~d}, J=7.71 \mathrm{~Hz}, 1 \mathrm{H}), 5.76(\mathrm{~d}, J=7.72 \mathrm{~Hz}, 1 \mathrm{H}), 5.52(\mathrm{~d}, J$ $=2.22 \mathrm{~Hz}, 1 \mathrm{H}), 3.96(\mathrm{~s}, 3 \mathrm{H}), 3.55(\mathrm{~s}, 3 \mathrm{H}), 1.31(\mathrm{~s}, 9 \mathrm{H}), 1.11(\mathrm{~s}, 9 \mathrm{H}) .{ }^{13} \mathrm{C} \mathrm{NMR}: \delta 198.1,164.0$, $163.7,145.1,143.9,142.7,135.1,130.0,129.2,128.3,127.9,126.9,126.3,125.1,123.4,109.3$, 104.3, 78.1, 53.2, 51.1, 34.6, 31.2, 29.4, 28.5. HRMS (EI) for $\mathrm{C}_{29} \mathrm{H}_{33} \mathrm{NO}_{6}$; Calcd. 491.2308; Found 491.2310.

Dimethyl-4,6-(t-butyl)-2-oxospiro[cyclohexa-2,4-diene-1,2'-[2H,11bH] [1,3]oxazino[2,3a]isoquinoline]-3',4'-dicarboxylate (21). Yellow semi-solid. IR(film) v $v_{\max }$ : 2955, 1742, 1708, 1667, 1592, 1566, 1430, 1276, 1230, $1150 \mathrm{~cm}^{-1} .{ }^{1} \mathrm{H}$ NMR: $\delta 7.25-7.13(\mathrm{~m}, 3 \mathrm{H}), 7.04(\mathrm{~d}, J=7.49$ $\mathrm{Hz}, 1 \mathrm{H}), 6.92(\mathrm{~s}, 1 \mathrm{H}), 6.54(\mathrm{~d}, J=1.29 \mathrm{~Hz}, 1 \mathrm{H}), 6.31(\mathrm{~d}, J=7.75 \mathrm{~Hz}, 1 \mathrm{H}), 5.95(\mathrm{~d}, J=1.31 \mathrm{~Hz}$, 1H), $5.73(\mathrm{~d}, J=7.74 \mathrm{~Hz}, 1 \mathrm{H}), 3.97(\mathrm{~s}, 3 \mathrm{H}), 3.55$ (s, 3H), $1.22(\mathrm{~s}, 9 \mathrm{H}), 1.14(\mathrm{~s}, 9 \mathrm{H}) .{ }^{13} \mathrm{C}$ NMR: $\delta$ 200.0, 164.1, 163.7, 144.3, 142.5, 136.2, 130.4, 129.9, 129.1, 127.8, 126.9, 126.3, 124.9, 123.7, 123.1, 115.8, 104.6, 78.6, 53.2, 51.3, 37.9, 35.7, 31.4, 28.3.HRMS (EI) for $\mathrm{C}_{29} \mathrm{H}_{33} \mathrm{NO}_{6}$; Calcd. 491.2308; Found 491.2314.

Dimethyl-2-oxospiro[acenaphthylene-1,2' [2H,11bH][1,3]oxazino[2,3a] isoquinoline] -3',4'dicarboxylate (2). Yellow crystalline solid; mp 155-157 ${ }^{\circ} \mathrm{C}$. IR(KBr) v $v_{\max }$ : 2955, 1748, 1728, 1700, 1593, 1573, 1431, 1290, 1222, $1148 \mathrm{~cm}^{-1} .{ }^{1} \mathrm{H}$ NMR: $\delta 8.05$ (q, $\left.J=7.53 \mathrm{~Hz}, 2 \mathrm{H}\right), 7.82(\mathrm{~d}, J$ $=8.16 \mathrm{~Hz}, 1 \mathrm{H}), 7.74(\mathrm{t}, J=7.59 \mathrm{~Hz}, 1 \mathrm{H}), 7.57-7.48(\mathrm{~m}, 2 \mathrm{H}), 7.26-7.03(\mathrm{~m}, 5 \mathrm{H}), 6.46(\mathrm{~d}, J=$ $7.75 \mathrm{~Hz}, 1 \mathrm{H}), 5.81(\mathrm{~d}, J=7.76 \mathrm{~Hz}, 1 \mathrm{H}), 3.99(\mathrm{~s}, 3 \mathrm{H}), 3.17(\mathrm{~s}, 3 \mathrm{H}) .{ }^{13} \mathrm{C} \mathrm{NMR}: \delta 200.8,163.6$, $163.5,145.1,143.0,138.4,131.4,130.9,130.5,129.7,129.4,128.5,128.1,127.9,127.0,126.2$, 125.6, 125.1, 123.2, 122.0, 119.8, 106.1, 105.0, 80.0, 53.3, 51.3. Anal. Calcd. for $\mathrm{C}_{27} \mathrm{H}_{19} \mathrm{NO}_{6}$; C, 71.52; H, 4.22; N, 3.09; Found C, 76.27; H, 4.44; N, 3.17.

Dimethyl-2-oxospiro[phenanthrene-1,2'-[2H,11bH][1,3]oxazino[2,3-a]isoquinoline]-3',4'-

dicarboxylate (23). Yellow crystalline solid; mp 202-204 ${ }^{\circ} \mathrm{C}$. IR(KBr) $v_{\max }$ : 2955, 1748, 1708, 1595, 1573, 1431, 1283, 1222, $1162 \mathrm{~cm}^{-1} .{ }^{1} \mathrm{H}$ NMR: $\delta 8.07(\mathrm{q}, J=4.56 \mathrm{~Hz}, 2 \mathrm{H}), 7.96(\mathrm{~d}, J=$ $7.69 \mathrm{~Hz}, 1 \mathrm{H}), 7.70(\mathrm{t}, J=7.67 \mathrm{~Hz}, 1 \mathrm{H}), 7.52(\mathrm{q}, J=7.05 \mathrm{~Hz}, 2 \mathrm{H}), 7.44-7.35(\mathrm{~m}, 2 \mathrm{H}), 7.16(\mathrm{t}, J$ $=7.42 \mathrm{~Hz}, 1 \mathrm{H}), 6.98(\mathrm{~d}, J=7.46 \mathrm{~Hz}, 2 \mathrm{H}), 6.63(\mathrm{~d}, J=7.57 \mathrm{~Hz}, 1 \mathrm{H}), 6.36(\mathrm{~d}, J=7.76 \mathrm{HZ}, 1 \mathrm{H})$, $6.23(\mathrm{~s}, 1 \mathrm{H}), 5.72(\mathrm{~d}, J=7.79 \mathrm{~Hz}, 1 \mathrm{H}), 4.03(\mathrm{~s}, 3 \mathrm{H}), 3.45(\mathrm{~s}, 3 \mathrm{H}) .{ }^{13} \mathrm{C} \mathrm{NMR}: \delta 193.5,163.7$, $162.9,145.2$, 137.3, 135.9, 134.2, 130.5, 129.2, 129.0, 128.7, 127.9, 127.8, 127.7, 127.4, 126.5, 
126.2, 124.9, 124.4, 124.2, 123.1, 122.6, 104.6, 103.8, 79.9, 52.7, 51.0. HRMS (EI) for $\mathrm{C}_{29} \mathrm{H}_{21} \mathrm{NO}_{6}$; Calcd. 479.1369; Found 479.1380.

Dimethyl-6-oxospiro[phenanthrene-1,2'-[2H,11bH][1,3]oxazino[2,3-a]isoquinoline]-3',4'dicarboxylate (24). Yellow crystalline solid; $m p$ 192-194 ${ }^{\circ} \mathrm{C}$. IR(KBr) $v_{\max }: 2955,1748,1708$, 1685, 1600, 1569, 1431, 1276, 1220, $1155 \mathrm{~cm}^{-1} .{ }^{1} \mathrm{H}$ NMR: $\delta 8.22(\mathrm{~d}, J=7.72 \mathrm{~Hz}, 1 \mathrm{H}), 7.96(\mathrm{dd}$, $J=8.00,11.78 \mathrm{~Hz}, 2 \mathrm{H}), 7.66(\mathrm{t}, J=7.65 \mathrm{~Hz}, 1 \mathrm{H}), 7.48-7.42(\mathrm{~m}, 2 \mathrm{H}), 7.36-7.23(\mathrm{~m}, 2 \mathrm{H}), 7.16(\mathrm{t}$, $J=7.39 \mathrm{~Hz}, 1 \mathrm{H}), 7.05-6.98(\mathrm{~m}, 3 \mathrm{H}), 6.77(\mathrm{~s}, 1 \mathrm{H}), 6.41(\mathrm{~d}, J=7.74 \mathrm{~Hz}, 1 \mathrm{H}), 5.75(\mathrm{~d}, J=7.74$ $\mathrm{Hz}, 1 \mathrm{H}), 4.00(\mathrm{~s}, 3 \mathrm{H}), 3.35(\mathrm{~s}, 3 \mathrm{H}) .{ }^{13} \mathrm{C} \mathrm{NMR}: \delta 194.7,163.8,163.6,145.1,137.3,136.9,134.8$, $131.2,129.8,129.3,129.3,129.2,128.5,128.2$, 127.7, 127.6, 126.9, 125.8, 125.0, 123.6, 123.4, 123.2, 109.5, 104.8, 79.2, 53.3, 51.6. HRMS (EI) for $\mathrm{C}_{29} \mathrm{H}_{21} \mathrm{NO}_{6}$; Calcd. 479.1369; Found 479.1302 .

Dimethyl-2-methoxy-3,5-di(t-butyl)-2-oxospiro[cyclohexa-2,4-diene-1,2'-[2H,11bH] [1,3]oxazino[2,3-a]isoquinoline]-3',4'-dicarboxylate (25). Yellow viscous liquid. IR(film) $v_{\max }$ : 2955, 1748, 1708, 1667, 1600, 1566, 1431, 1270, $1148 \mathrm{~cm}^{-1} .{ }^{1} \mathrm{H}$ NMR: $\delta$ 7.28-7.12 (m, 3H), 7.04 $(\mathrm{d}, J=8.70 \mathrm{~Hz}, 2 \mathrm{H}), 6.62(\mathrm{~s}, 1 \mathrm{H}), 6.33(\mathrm{~d}, J=7.71 \mathrm{~Hz}, 1 \mathrm{H}), 5.72(\mathrm{~d}, J=7.72 \mathrm{~Hz}, 1 \mathrm{H}), 4.03(\mathrm{~s}$, $3 \mathrm{H}), 3.58(\mathrm{~s}, 3 \mathrm{H}), 3.54(\mathrm{~s}, 3 \mathrm{H}), 1.29(\mathrm{~s}, 9 \mathrm{H}), 1.21(\mathrm{~s}, 9 \mathrm{H}) .{ }^{13} \mathrm{C} \mathrm{NMR}: \delta 195.9,163.8,163.6$, $154.6,144.9,141.3,138.7,138.4,130.2$, 129.3, 128.6, 127.7, 126.9, 126.1, 124.9, 123.6, 109.5, 104.2, 78.6, 59.5, 53.1, 51.2, 34.6, 34.5, 30.0, 29.8. HRMS (EI) for $\mathrm{C}_{30} \mathrm{H}_{35} \mathrm{NO}_{7}$; Calcd. 521.2413; Found 521.2452.

Dimethyl-3-(t-butyl)-6-oxospiro[cyclohexa-2,4-diene-1,2'-[2H,11bH] [1,3]oxazino[2,3a]isoquinoline]-3',4'-dicarboxylate (26). Yellow solid; mp 96-98 ${ }^{\circ} \mathrm{C}$. IR(KBr) $v_{\max }$ : 2962, 1741, 1708, 1667, 1593, 1566, 1430, 1276, 1236, $1148 \mathrm{~cm}^{-1} .{ }^{1} \mathrm{H}$ NMR: $\delta 7.31-7.17$ (m, 3H), 7.08 (d, $J$ $=7.38 \mathrm{~Hz}, 1 \mathrm{H}), 6.87(\mathrm{~s}, 1 \mathrm{H}), 6.46(\mathrm{~d}, J=9.89 \mathrm{~Hz}, 1 \mathrm{H}), 6.35(\mathrm{~d}, J=7.71 \mathrm{~Hz}, 1 \mathrm{H}), 6.01(\mathrm{~s}, 1 \mathrm{H})$, $5.89(\mathrm{~d}, J=9.87 \mathrm{~Hz}, 1 \mathrm{H}), 5.79(\mathrm{~d}, J=7.73 \mathrm{~Hz}, 1 \mathrm{H}), 3.97(\mathrm{~s}, 3 \mathrm{H}), 3.58(\mathrm{~s}, 3 \mathrm{H}), 1.18(\mathrm{~s}, 9 \mathrm{H}) .{ }^{13} \mathrm{C}$ NMR: $\delta$ 198.9, 163.4, 163.0, 144.4, 141.5, 138.7, 130.6, 129.9, 129.3, 128.2, 127.1, 126.3, 125.7, 124.8, 123.2, 118.4, 108.3, 104.9, 78.8, 53.1, 51.3, 35.4, 28.2. HRMS (EI) for $\mathrm{C}_{25} \mathrm{H}_{25} \mathrm{NO}_{6}$; Calcd. 435.1682; Found 435.1688.

Dimethyl-4-(t-butyl)-6-oxospiro[cyclohexa-2,4-diene-1,2'-[2H,11bH] [1,3]oxazino[2,3a]isoquinoline]-3',4'-dicarboxylate(27). Yellow viscous liquid. IR (film) $v_{\max }$ : 2955, 1743, 1712, 1672, 1597, 1565, 1434, $1287 \mathrm{~cm}^{-1} .{ }^{1} \mathrm{H}$ NMR: $\delta 7.29-7.16(\mathrm{~m}, 3 \mathrm{H}), 7.06(\mathrm{~d}, J=7.50 \mathrm{~Hz}$, $1 \mathrm{H}), 6.65(\mathrm{~d}, J=10.08 \mathrm{~Hz}, 1 \mathrm{H}), 6.43(\mathrm{~d}, J=10.04 \mathrm{~Hz}, 1 \mathrm{H}), 6.34(\mathrm{~d}, J=7.73 \mathrm{~Hz}, 1 \mathrm{H}), 6.25(\mathrm{~d}, J$ $=9.56 \mathrm{~Hz}, 1 \mathrm{H}), 5.98(\mathrm{~s}, 1 \mathrm{H}), 5.75(\mathrm{~d}, J=7.75 \mathrm{~Hz}, 1 \mathrm{H}), 3.94(\mathrm{~s}, 3 \mathrm{H}), 3.56(\mathrm{~s}, 3 \mathrm{H}), 1.21(\mathrm{~s}, 9 \mathrm{H})$. ${ }^{13} \mathrm{C}$ NMR: $\delta$ 195.8, 164.0, 163.4, 144.9, 138.6, 135.3, 130.1, 129.5, 128.2, 127.1, 126.8, 125.9, 125.4, 124.1, 123.8, 119.2, 118.4, 104.6, 79.7, 53.2, 51.5, 35.3, 28.2. HRMS (EI) for $\mathrm{C}_{25} \mathrm{H}_{25} \mathrm{NO}_{6}$; Calcd. 435.1682; Found 435.1673.

Dimethyl-3-methyl-4-oxospiro[cyclohexa-2,4-diene-1,2'-[2H,11bH] [1,3]oxazino[2,3a]isoquinoline]-3',4'-dicarboxylate (28). Yellow crystalline solid; mp 195-197 ${ }^{\circ} \mathrm{C}$. IR (KBr) $v_{\max }: 2955,1742,1708,1670,1647,1593,1566,1431,1276,1236,1155 \mathrm{~cm}^{-1} .{ }^{1} \mathrm{H}$ NMR: $\delta 7.32-$ $7.21(\mathrm{~m}, 3 \mathrm{H}), 7.16-7.09(\mathrm{~m}, 2 \mathrm{H}), 6.36(\mathrm{~d}, J=7.71 \mathrm{~Hz}, 1 \mathrm{H}), 6.29-6.20(\mathrm{~m}, 3 \mathrm{H}), 5.82(\mathrm{~d}, J=$ $7.74 \mathrm{~Hz}, 1 \mathrm{H}), 3.95(\mathrm{~s}, 3 \mathrm{H}), 3.58(\mathrm{~s}, 3 \mathrm{H}), 1.85$ (s, 3H). ${ }^{13} \mathrm{C} \mathrm{NMR}: \delta$ 185.4, 163.7, 163.1, 156.6, 
$148.4,145.5,143.3,129.7,129.6,129.1,128.5,127.8,127.7,127.5,127.4,127.2,125.8,125.5$, 125.3, 123.6,123.4, 109.0, 105.0, 79.4, 53.3, 51.7, 17.7. Anal. Calcd. for $\mathrm{C}_{22} \mathrm{H}_{19} \mathrm{NO}_{6} ; \mathrm{C}, 67.17$; H, 4.87; N, 3.56; Found C, 67.27; H, 4.64; N, 3.42.

Dimethyl-2-methyl-4-oxospiro[cyclohexa-2,4-diene-1,2'-[2H,11bH] [1,3]oxazino[2,3-a]isoquinoline]-3',4'-dicarboxylate (29). Yellow crystalline solid; mp 184-186 ${ }^{\circ} \mathrm{C}$. IR(KBr) $v_{\max }$ : 2955, 1742, 1715, 1670, 1647, 1593, 1566, 1431, $1236 \mathrm{~cm}^{-1} .{ }^{1} \mathrm{H}$ NMR: $\delta$ 7.38-7.22 (m, 3H), 7.11 $(\mathrm{d}, J=7.56 \mathrm{~Hz}, 1 \mathrm{H}), 6.87(\mathrm{~s}, 1 \mathrm{H}), 6.47(\mathrm{dd}, J=3.03,9.82 \mathrm{~Hz}, 1 \mathrm{H}), 6.34(\mathrm{~d}, J=7.96 \mathrm{~Hz}, 1 \mathrm{H})$, $6.28(\mathrm{~d}, J=6.26 \mathrm{~Hz}, 1 \mathrm{H}), 6.23(\mathrm{~d}, J=4.38 \mathrm{~Hz}, 1 \mathrm{H}), 5.80(\mathrm{~d}, J=7.75 \mathrm{~Hz}, 1 \mathrm{H}), 3.95(\mathrm{~s}, 3 \mathrm{H}), 3.58$ (s, 3H), 1.93 (s, 3H). ${ }^{13} \mathrm{C}$ NMR: $\delta 186.1,163.8,163.4,147.3,144.8,142.7,138.5,137.1,135.1$, $130.1,129.7,129.6,128.3,127.7,127.3,127.2,125.9,125.4,123.3,107.4,104.9,79.3,53.3$, 51.75, 15.9. Anal. Calcd. for $\mathrm{C}_{22} \mathrm{H}_{19} \mathrm{NO}_{6} ; \mathrm{C}, 67.17$; H, 4.87; N, 3.56; Found C, 67.11; H, 4.749; N, 3.48 .

Dimethyl-3-phenyl-4-oxospiro[cyclohexa-2,4-diene-1,2'-[2H,11bH] [1,3]oxazino[2,3-a]isoquinoline]-3',4'-dicarboxylate (30). Yellow crystalline solid; mp 175-177 ${ }^{\circ} \mathrm{C}$. IR (KBr) $v_{\max }$ : 2955, 1742, 1708, 1670, 1600, 1566, 1431, 1270, 1235, $1148 \mathrm{~cm}^{-1} .{ }^{1} \mathrm{H}$ NMR: $\delta$ 7.45-7.20 (m, $5 \mathrm{H}), 7.13(\mathrm{t}, J=8.10 \mathrm{~Hz}, 2 \mathrm{H}), 7.01(\mathrm{~d}, J=7.47 \mathrm{~Hz}, 1 \mathrm{H}), 6.94(\mathrm{~d}, J=7.58 \mathrm{~Hz}, 1 \mathrm{H}), 6.40-6.28(\mathrm{~m}$, $3 \mathrm{H}), 6.17(\mathrm{~d}, J=1.64 \mathrm{~Hz}, 1 \mathrm{H}), 5.73(\mathrm{~d}, J=7.73 \mathrm{~Hz}, 1 \mathrm{H}), 5.48(\mathrm{~s}, 1 \mathrm{H}), 3.96(\mathrm{~s}, 3 \mathrm{H}), 3.71(\mathrm{~s}, 3 \mathrm{H})$.

${ }^{13} \mathrm{C}$ NMR: $\delta$ 186.0, 163.2, 163.1, 156.4, 149.1, 144.9, 142.0, 138.0, 129.5, 129.1, 128.9, 128.6, $128.5,128.1,127.9,127.7,127.3,127.1,125.4,123.1,105.0,79.6,53.4,52.0$. HRMS (EI) for $\mathrm{C}_{27} \mathrm{H}_{21} \mathrm{NO}_{6}$; Calcd. 455.1369; Found 455.1349.

Dimethyl-2-phenyl-4-oxospiro[cyclohexa-2,4-diene-1,2'-[2H,11bH] [1,3]oxazino[2,3-a]isoquinoline]-3',4'-dicarboxylate (31). Yellow crystalline solid; mp 162-164 ${ }^{\circ} \mathrm{C}$. IR( $\left.\mathrm{KBr}\right) v_{\max }$ : 2955, 1742, 1708, 1667, 1593, 1566, 1431, 1276, 1236, $1148 \mathrm{~cm}^{-1} .{ }^{1} \mathrm{H}$ NMR: $\delta$ 7.40-7.14 (m, $10 \mathrm{H}), 6.37(\mathrm{~d}, J=1.77 \mathrm{~Hz}, 1 \mathrm{H}), 6.31(\mathrm{dd}, J=1.75,10.02 \mathrm{~Hz}, 1 \mathrm{H}), 6.19(\mathrm{~s}, 1 \mathrm{H}), 6.05(\mathrm{~d}, J=$ $7.71 \mathrm{~Hz}, 1 \mathrm{H}), 5.80(\mathrm{~d}, J=7.73 \mathrm{~Hz}, 1 \mathrm{H}), 3.72(\mathrm{~s}, 3 \mathrm{H}), 3.60(\mathrm{~s}, 3 \mathrm{H}) .{ }^{13} \mathrm{C} \mathrm{NMR}: \delta 185.9,163.7$, $162.7,157.8,145.0,143.3,136.9,130.0,129.8,129.7,128.7,128.2,128.1,127.7,127.3,127.2$, 125.6, 125.4, 123.7, 110.3, 104.7, 79.6, 53.1, 51.7. HRMS (EI) for $\mathrm{C}_{27} \mathrm{H}_{21} \mathrm{NO}_{6}$; Calcd. 455.1369; Found 455.1315.

Dimethyl-4-oxospiro[cyclohexa-2,4-diene-1,2'-[2H,11bH][1,3]oxazino[2,3-a] isoquinoline]3',4'-dicarboxylate (32). Yellow crystalline solid; mp 167-169 ${ }^{\circ} \mathrm{C}$. IR(KBr) $v_{\max }$ : 2956, 1742, 1702, 1672, 1587, 1562, 1427, 1273, $1233 \mathrm{~cm}^{-1} .{ }^{1} \mathrm{H}$ NMR: $\delta$ 7.37-7.25 (m, 3H), 7.14-7.06 (m, 2H), $6.51(\mathrm{dd}, J=2.94,9.92 \mathrm{~Hz}, 1 \mathrm{H}), 6.36-6.25(\mathrm{~m}, 4 \mathrm{H}), 5.82(\mathrm{~d}, J=7.74 \mathrm{~Hz}, 1 \mathrm{H}), 3.96(\mathrm{~s}, 3 \mathrm{H})$, 3.59 (s, 3H). ${ }^{13} \mathrm{C}$ NMR: $\delta$ 185.4, 163.7, 163.1, 147.6, 145.3, 143.1, 130.3, 129.9, 129.6, 128.4, $127.7,127.4,125.8,125.5,123.2,113.7,111.8,105.2,85.9,53.4,51.8$. HRMS (EI) for $\mathrm{C}_{21} \mathrm{H}_{17} \mathrm{NO}_{6}$; Calcd. 379.1056; Found 379.1102.

Dimethyl-1,2-dihydro-2-oxo-1-phenylspiro[3H-indole-3,2'-[2H,11bH] [1,3]oxazino[2,3-a] isoquinoline]-3',4'-dicarboxylate (33). Yellow crystalline solid; mp 240-242 ${ }^{\circ} \mathrm{C}$. $\mathrm{IR}(\mathrm{KBr}) v_{\max }$ : 2955, 1741, 1711, 1620, 1580, 1501, 1426, 1276, $1145 \mathrm{~cm}^{-1} .{ }^{1} \mathrm{H}$ NMR: $\delta$ 7.57-7.48 (m, 4H), 7.44-7.38 (m, 2H), 7.26-7.14 (m, 5H), $7.08(\mathrm{~d}, \mathrm{~J}=7.54 \mathrm{~Hz}, 1 \mathrm{H}), 6.99(\mathrm{t}, \mathrm{J}=7.18 \mathrm{~Hz}, 1 \mathrm{H}), 6.79$ $(\mathrm{d}, \mathrm{J}=7.80 \mathrm{~Hz}, 1 \mathrm{H}), 6.42$ (d, J = 7.73 Hz, 1H), 5.83 (d, J = 7.74 Hz, 1H), 3.98 (s, 3H), 3.53 (s, 
$3 \mathrm{H}) .{ }^{13} \mathrm{C}$ NMR: $\delta 173.3,163.1,162.9,144.8,144.7,133.8,129.5,129.2,129.1,128.9,127.7$, 127.6, 127.5, 126.6, 125.9, 125.5, 124.6, 123.2, 122.8, 122.5, 109.0, 105.1, 104.7, 79.1, 52.8, 51.2. Anal. Calcd. for $\mathrm{C}_{29} \mathrm{H}_{22} \mathrm{~N}_{2} \mathrm{O}_{6}$ : C, 70.44; H, 4.48; N, 5.67; Found C, 70.80; H, 4.62; N, 5.51.

Dimethyl-1,2-dihydro-2-oxo-1-methylspiro[3H-indole-3,2'-[2H,11bH] [1,3]oxazino[2,3-a] isoquinoline]-3',4'-dicarboxylate (34). Yellow crystalline solid; mp $210-212{ }^{\circ} \mathrm{C}$. $\mathrm{IR}(\mathrm{KBr}) v_{\max }$ : 2955, 1742, 1721, 1647, 1593, 1566, 1431, 1276, $1155 \mathrm{~cm}^{-1} .{ }^{1} \mathrm{H}$ NMR: $\delta 7.36$ (d, $J=7.48 \mathrm{~Hz}$, 1H), 7.29-7.16 (m, 4H), 7.08 (t, $J=7.57 \mathrm{~Hz}, 2 \mathrm{H}), 6.96(\mathrm{t}, J=7.47 \mathrm{~Hz}, 1 \mathrm{H}), 6.80(\mathrm{~d}, J=7.74 \mathrm{~Hz}$, $1 \mathrm{H}), 6.39(\mathrm{~d}, J=7.73 \mathrm{~Hz}, 1 \mathrm{H}), 5.81(\mathrm{~d}, J=7.74 \mathrm{~Hz}, 1 \mathrm{H}), 3.97(\mathrm{~s}, 3 \mathrm{H}), 3.46(\mathrm{~s}, 3 \mathrm{H}), 3.28(\mathrm{~s}, 3 \mathrm{H})$. ${ }^{13} \mathrm{C}$ NMR: $\delta$ 174.5, 163.4, 163.4, 145.3, 145.2, 130.1, 129.8, 129.4, 128.3, 127.1, 126.2, 125.1, $123.4,123.2,122.9,120.4,108.2,105.7,105.2,79.6,53.3,51.7,26.3$. HRMS (EI) for $\mathrm{C}_{24} \mathrm{H}_{20} \mathrm{~N}_{2} \mathrm{O}_{6}$; Calcd. 432.1321; Found 432.1322.

Dimethyl-1,2-dihydro-2-oxo-1-benzylspiro[3H-indole-3,2'-[2H,11bH] [1,3]oxazino[2,3-a]isoquinoline]-3',4'-dicarboxylate (35). Yellow crystalline solid; mp $235-237{ }^{\circ} \mathrm{C}$. IR( $\left.\mathrm{KBr}\right) v_{\max }$ : 2955, 1742, 1715, 1600, 1565, 1431, 1351, 1222, 1148, $1000 \mathrm{~cm}^{-1} .{ }^{1} \mathrm{H}$ NMR: $\delta$ 7.43-7.06 (m, 9H), 6.92 (t, $J=7.47 \mathrm{~Hz}, 1 \mathrm{H}), 6.71(\mathrm{~d}, J=7.74 \mathrm{~Hz}, 1 \mathrm{H}), 6.40(\mathrm{~d}, J=7.72 \mathrm{~Hz}, 1 \mathrm{H}), 5.81(\mathrm{~d}, J=$ $7.74 \mathrm{~Hz}, 1 \mathrm{H}), 5.28(\mathrm{~s}, 1 \mathrm{H}), 5.09$ (d, $J=15.58 \mathrm{~Hz}, 1 \mathrm{H}), 4.83(\mathrm{~d}, J=15.59 \mathrm{~Hz}, 1 \mathrm{H}), 3.98(\mathrm{~s}, 3 \mathrm{H})$, $3.30(\mathrm{~s}, 3 \mathrm{H}), 2.81(\mathrm{~s}, 2 \mathrm{H}) .{ }^{13} \mathrm{C}$ NMR: $\delta$ 174.6, 164.8, 163.5, 145.5, 144.3, 135.8, 130.0, 129.8, $129.5,128.8,128.6,128.2,127.7,127.6,127.1,126.3,125.2,123.5,123.2,123.0,109.2$, 105.2 , 104.9, 79.5, 53.3, 51.5, 43.9. HRMS (EI) for $\mathrm{C}_{30} \mathrm{H}_{24} \mathrm{~N}_{2} \mathrm{O}_{6}$; Calcd. 508.1638; Found 508.1673.

\section{Acknowledgements}

S.A.R., A. N. and B.A.T thank Council of Scientific and Industrial Research, New Delhi for financial assistance.

\section{References}

1. Hetero Diels-Alder Methodology in Organic Synthesis; Boger, D. L.; Weinreb, S. M., Eds.; Academic Press: San Diego, 1987.

2. (a) Huisgen, R. In Topics in Heterocyclic Chemistry; Castle, R., Ed; John Wiley \& Sons: New York, 1969, Chapter 8, p 223. (b) Huisgen, R., Ger. Z. Chem. 1968, 8, 290.

3. (a) Padwa, A.; Harring S.R.; Semones M.A. J. Org. Chem. 1998, 63, 44. (b) Padwa, A.; Harring S.R.; Semones M.A. J. Org. Chem. 1995, 60, 2952.

4. (a) Swenton, J. S.; Jackson, D. K.; Narasimhan, L. J. Am. Chem. Soc. 1979, 101, 3989. (b) Bach, R. D.; Wendt, J. A.; Gauvreau, P. J. J. Am. Chem. Soc. 1994, 116, 9921. (c) Mann, A.; Ungureanu, I.; Klotz, P.; Schoenfelder, A. Chem. Commun. 2001, 958.

5. (a) Schaumann, E; Kausch, E; Walter, W. Chem. Ber. 1974, 107, 3574. (b) Huisgen, R. Liebigs Ann. Chem. 1965, 688, 98. (c) Butler, G. B.; Turner, S. R.; Guilbault, L. J. J. Org. 
Chem. 1971, 36, 2838. (d) Knölker, H. J.; Boese, R. J. Chem. Soc., Chem. Commun. 1988, 1151.

6. Huisgen, R.; Morikawa, M.; Herbig, K.; Brunn, E. Chem. Ber. 1967, 100, 1094.

7. (a) Nair, V.; Vinod, A. U.; Chem. Comm. 2000, 1019. (b) Nair, V.; Vinod A. U.; Rajesh, C. J. Org. Chem. 2001, 66, 4427. (c) Nair, V.; Bindu, S.; Balagopal, L. Tetrahedron Lett. 2001, 42, 2043.

8. (a) Nair, V.; Sreekanth A. R.; Abhilash, N.; Bhadbhadhe, M. M.; Gonnade, R. C. Org. Lett. 2002, 4, 3575. (b) Nair, V.; Sreekanth, A. R.; Biju, A. T. and Rath, N. P. Tetrahedron Lett. 2003, 44, 729.

9. (a) Nair, V.; Vinod, A. U.; Nair, J. S.; Sreekanth, A. R.; Rath, N. P. Tetrahedron Lett. 2000, 41, 6675. (b) Nair, V.; Nair, J. S.; Vinod, A. U. Synthesis 2000, 1713. (c) Nair, V.; Sheela, K. C.; Rath, N. P.; Eigendorf, G. K. Tetrahedron Lett. 2000, 41, 6217.

10. (a) Campagna, F.; Carotti, A.; Casini, G.; Otto, A. M. Farmaco 1995, 50, 137. (b) Bernath, G.; Fulop, F.; Kobor, J. Acta Pharm. Hung. 1993, 63, 129. (c) Gabor, B.; Kobor, J.; Fulop, F.; Sohar, P.; Perjesi, P.; Ezer, E.; Hajos, G.; Palosi, E.; Denes, L.; Szporny, L. Ger. Offen. 1985, 39 pp. CODEN: GWXXBX DE 3439131 A1. 